\title{
Genomically-Directed Monotherapy
}

National Cancer Institute

\section{Source}

National Cancer Institute. Genomically-Directed Monotherapy. NCI Thesaurus. Code C121155.

The use of genomic sequencing to direct cancer therapy. A profile of the DNA and RNA expressed by a patient's tumor is obtained and used to select known therapies that will exploit the specific genetic defects found in that tumor. 